\title{
Chronic hepatitis C - what do the new drugs offer and who should get them first?
}

\author{
Author: Stephen D Ryder ${ }^{\mathrm{A}}$
}

\begin{abstract}
Until recently in the UK the treatment of HCV depended on combination regimes of interferon (IFN) and the antiviral drug ribavirin. These regimes required regular injections and were of variable duration (generally for a minimum of 12 weeks), and the use of IFN often caused unacceptable side effects (thrombocytopenia, leukopenia and depression). Of the common HCV genotypes in the UK, genotype 1 responded relatively poorly to these regimes $(50-60 \%$ viral clearance), while most ( $80 \%$ plus) of genotype 3 patients responded with sustained viral clearance. Patients with severe liver disease (decompensated cirrhosis) tolerated these regimens very poorly and often their liver function deteriorated. The recent introduction of a series of direct anti-viral agents (DAAs) offers the potential to revolutionise treatment, particularly in genotype 1 patients and those with advanced liver disease, as drug regimens avoiding IFN have been developed, and can be curative in, for example, $95 \%$ of genotype 1 patients. The DAAs are currently being evaluated and introduced into UK clinical practice. Choice of drug regime, and strategies for identifying patient groups suitable for treatment, are discussed, as are the prospects for eventual complete control of the HCV epidemic.
\end{abstract}

\section{Introduction}

We are in the midst of a revolution in hepatitis $\mathrm{C}$ treatments as we move from modestly effective regimes with significant side effects, to short duration all-oral treatments with cure rates in excess of $95 \%$. This has been achieved in less than 25 years from discovery of the hepatitis $\mathrm{C}$ virus (HCV), and represents a major triumph for traditional drug discovery. Academics and the pharmaceutical industry have benefitted from two major advantages. First, HCV was the first human pathogen to be discovered by detection of its genome in the serum of sufferers, and second, the previous identification of other flaviviruses gave significant clues as to targets for drug development.

Author: ${ }^{\text {A }}$ consultant hepatologist, Nottingham University Hospitals NHS Trust and Biomedical Research Unit, Queens Medical Centre, Nottingham, UK
Knowledge of the structure and function of viral proteins was an essential first step and a number of key proteins and synthetic steps were identified. Key among those were the HCV protease and polymerase, both vital in virion production. ${ }^{1}$ The first proof of concept came in 2003 with a small molecule from Bayer which inhibited the HCV protease in vitro and produced a significant short-term fall in viraemia in vivo. A major boost to drug development came from development of the replicon system in the 2000s which allowed direct testing of the power of small molecules to block HCV replication in a laboratory setting. ${ }^{2}$ These developments led to an explosion of new agents under test, targeting every phase of $\mathrm{HCV}$ replication, from entry into the hepatocyte to assembly and virion release. Key targets that emerged were: the HCV protease (NS3/4) and the polymerase (NS5 A/B).

It was quickly shown that a single agent would not produce sustained viral suppression. Virion production in HCV is very high, around $10^{12}$ per day, implying every mutation in its genome will occur in every patient every day and hence use of drugs with a single block point will allow the rapid development of resistance. This led to the development of combinations of drugs, initially direct-acting antivirals (DAAs) with interferon (IFN) and ribavirin (RBV), and then combinations of DAAs without IFN.

The principle behind combining the DAAs is to combine the most potent drugs and those with the highest barrier to resistance. Since different HCV genotypes are structurally very diverse, single oral agents have different levels of effectiveness against different block points, the regimes for different genotypes vary substantially, both in choice of drug combination and efficacy. The concept is not new; we have known for many years that IFN-based therapies differ in their effect and required duration of treatment by $\mathrm{HCV}$ genotype. The change is that with the new DAAs, genotype 1 (G1) (the more difficult to treat with IFN-based therapy) is more susceptible to treatment, and genotype 3 (G3) (a significant population in the UK) is the new difficult to treat genotype.

\section{Emerging drugs}

At present, there are a number of drugs licenced for use in $\mathrm{HCV}$ and, from a UK perspective, have National Institute for Health and Care Excellence (NICE) approval or are likely to gain it in the next few months. 


\begin{tabular}{|c|c|}
\hline HCV genotype & Adult patient population \\
\hline \multirow[t]{2}{*}{1} & Treatment naïve ${ }^{a}$ \\
\hline & Treatment experienced ${ }^{a}$ \\
\hline \multirow[t]{2}{*}{3} & Treatment naïve with cirrhosis ${ }^{a}$ \\
\hline & Treatment experienced $^{a}$ \\
\hline 4,5 or 6 & $\begin{array}{l}\text { Treatment naïve and experienced with } \\
\text { cirrhosis }^{a}\end{array}$ \\
\hline
\end{tabular}

${ }^{a}$ interferon eligible. $\mathrm{HCV}=$ hepatitis $\mathrm{C}$ virus; Peg-IFN = pegylated interferon; $\mathrm{RBV}=$ ribavirin .

\section{Sofosbuvir}

Sofosbuvir is a DNA polymerase inhibitor and has been recommended by NICE for different genomes either in combination with pegylated (Peg)-IFN and RBV (Table 1), or in combination with RBV alone (Table 2). The precise NICE guidance for its use is can be accessed at http://www.england. nhs.uk/commissioning/spec-services/npc-crg/group-a/a02/.

\section{Simeprevir}

Simeprevir (a second generation protease inhibitor) is also available for use in combination with IFN and RBV for G1 patients. It is interesting that it will essentially replace boceprevir and telaprevir, drugs that were only introduced within the last few years, given the shorter duration therapy and better side effect profile. The NHS commissioning policy for simeprevir can be found at http://www.england.nhs.uk/ commissioning/spec-services/npc-crg/group-a/a02/

\section{GI patients}

The above regimes will change very shortly, particularly for G1 patients where two all-oral combination treatments are available and going through NICE approval. One is a combination of the DNA polymerase inhibitor sofosbuvir, with an NS5A protease inhibitor ledipasvir, taken as a once-aday single pill (Harvoni), and the second is a combination of four drugs from AbbVie (the NS5A inhibitor ombitasvir, the NS3/4A protease inhibitor paritaprevir, and the HIV protease inhibitor Norvir (ritonavir), with a non-nucleoside NS5B palm polymerase inhibitor dasabuvir) marketed as VIEKIRAX ${ }^{\circledR}$

\section{Table 2. Sofosbuvir in combination with RBV.}

\begin{tabular}{|c|c|}
\hline HCV genotype & Adult patient population \\
\hline \multirow[t]{2}{*}{2} & Treatment-naïve \\
\hline & Treatment-experienced ${ }^{a}$ \\
\hline \multirow[t]{2}{*}{3} & Treatment-naïve with cirrhosis $^{b}$ \\
\hline & Treatment-experienced with cirrhosis \\
\hline
\end{tabular}

(ombitasvir/paritaprevir/ritonavir tablets) + EXVIERA ${ }^{\circledR}$ (dasabuvir tablets).

These regimens are highly potent with sustained virologic response (SVR) rates in G1 in excess of 95\%; essentially, these regimes cure. Very rarely patients relapse, ie a very small number of patients become HCV RNA negative on therapy, do not clear the virus completely and HCV RNA returns in their blood within 12 weeks of stopping treatment. Interestingly this is not due to drug-resistance; if re-exposed to the same agents the virus will again be suppressed and a longer duration of therapy can produce cure.

\section{G3 patients}

The transition to all-oral regimens in G3 patients is not yet imminent. The AbbVie regimen is not very effective against G3. However, combinations such as daclatasvir/sofosbuvir or Harvoni have SVR rates in excess of $90 \%{ }^{4}$ This failure rate is higher in those with advanced liver disease, and NHS England has recommended that patients with cirrhosis and liver failure are treated with either daclatasvir, sofosbuvir and RBV (12 weeks), or sofosbuvir, ledipasvir and ribavirin for 12 weeks.

\section{Decompensated patients}

The move to all-oral therapy, without IFN, is a major advance notably for patients with liver failure who cannot safely be treated with IFN-containing regimens. NHS England have funded treatment in 700 patients with decompensated cirrhosis using all-oral treatment. The results are not yet available but there is optimism that cure of HCV will improve liver function and avoid liver deaths in this group.

\section{Future treatment trends}

As we move into an era where IFN is dropped from the treatment algorithm for HCV, various factors impact on who will be treated with what and when. While all of these treatments are likely to be highly cost effective, given they produce a cure and avoid long-term risks of liver failure and cancer, they are expensive. A treatment of 12 weeks with

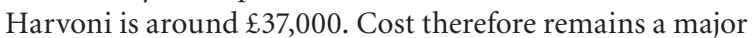
barrier to access and sofosbuvir has not been approved for use in some countries simply because of the financial burden. Cost

\section{Key points}

New therapies for HCV are oral with no major side effects

These therapies will cure $>90 \%$ of patients

$\mathrm{G} 1$ responds to the therapies better than $\mathrm{G} 3$

Case finding in primary care will be key to reducing the burden of liver disease due to HCV

It is possible to eradicate HCV by 2030 if we increase diagnosis and treatment rates by 2.5 fold

KEYWORDS: Hepatitis C, therapy, risk factors, cirrhosis, HCV genotype, sofosbuvir, simeprevir, interferon alpha 


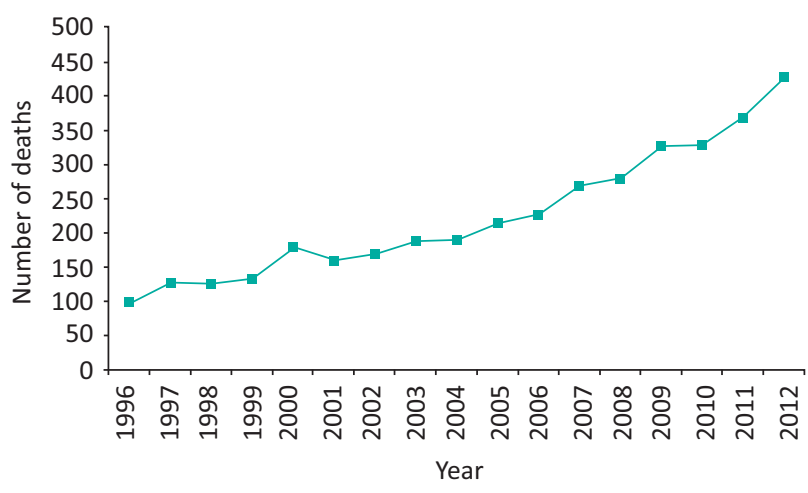

Fig 1. Increase in mortality from hepatitis C in the UK from 1996 to 2012. Deaths from end-stage liver disease (defined by codes or text entries for ascites, bleeding esophageal varices, hepato-renal sydrome, hepatic encephalopathy or hepatic failure) or hepatocellular carcinoma in those with hepatitis $\mathrm{C}$ mentioned on the death certificate. Reproduced with permission. ${ }^{5}$

will fall as competitors enter the market and many patients may be effectively treated with very short duration regimens: 8 weeks for G1 patients with mild liver disease with its resulting one-third reduction in cost per patient. This combination of cost and lack of side effects will help determine who can access treatment at what time.

On a population basis, the aims of treating and curing HCV are to stop the rising number of deaths from hepatitis $\mathrm{C}$ (Fig 1) and then potentially eradicating it as a significant health problem.
From a UK perspective, around $60 \%$ of all HCV-positive people have been probably been identified. ${ }^{5}$ Individuals with HCV infection comprise three key populations.

> Individuals known to be HCV positive and are in regular contact with secondary care treatment services; this group includes those who have received previous treatment regimens which have failed and those who, in the knowledge of better treatments, have elected to delay therapy.

> High-risk populations where more systematic testing regimens are being introduced, with 'opt-out' testing in drug treatment services, and in the prison service where a significant number of people who inject drugs are identified. ${ }^{5}$

$>$ The most difficult to reach group: individuals who are unaware of their HCV status and whose risk factor may have been many years ago.

Different strategies will be required to access these populations. For patients known to services, treatment is likely to be prioritised on the basis of clinical need, as assessed by severity of liver disease. People with cirrhosis are at relatively short-term risk of problems and therefore would access the new therapies first. This is a highly cost-effective group to treat irrespective of genotype. There will also be people with other factors which would affect the risks to them (young women wanting a family who want to be certain the small risk of vertical transmission is abolished, pre-surgical treatment, renal failure, contact sports, those at a high risk of progression from mild to more severe liver disease such as HIV co-infection or post liver transplantation). Prioritising one group over another for access would be difficult and fraught with challenge. Given the current restrictions via NICE and time frame, together with the fact
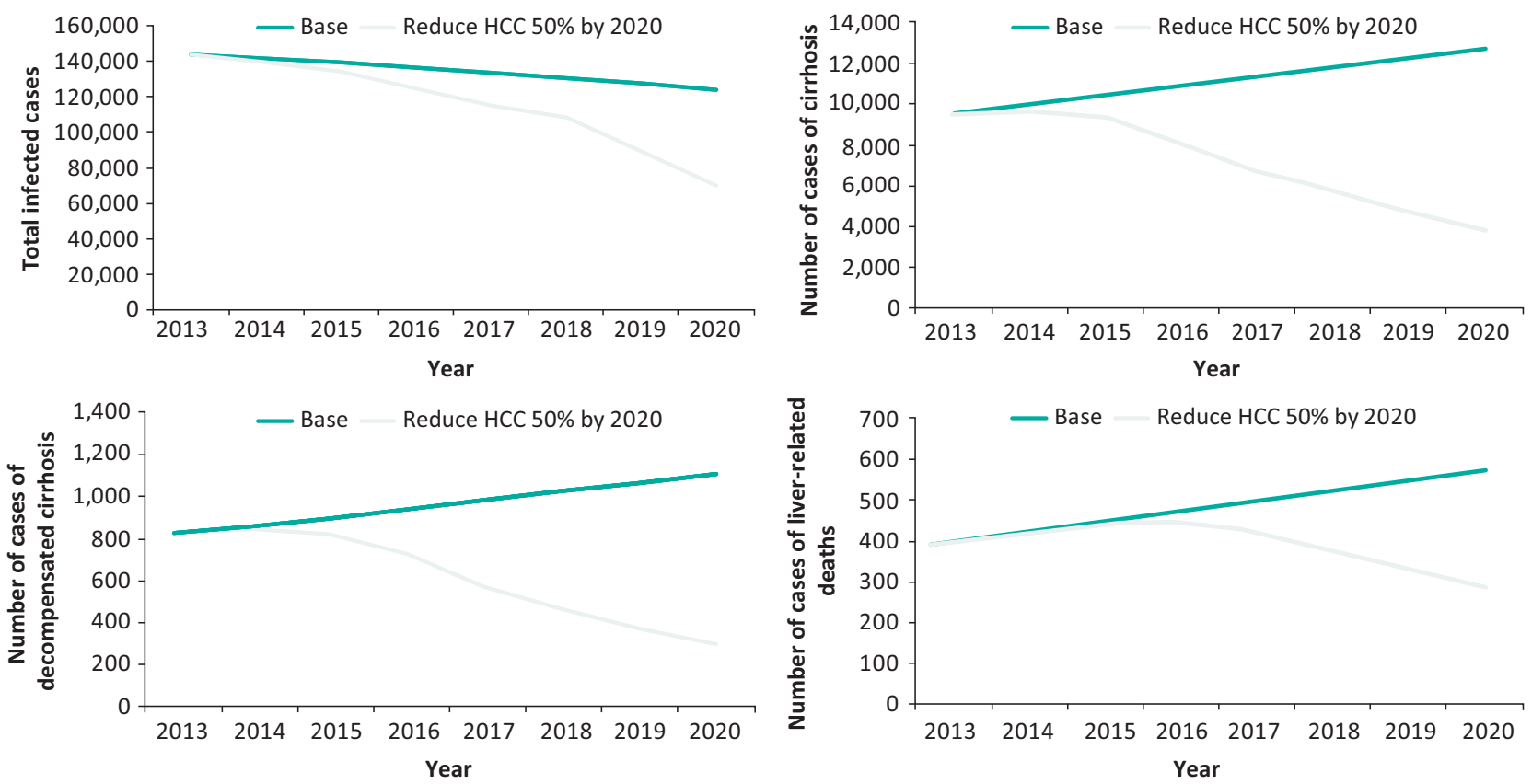

Fig 2. The impact of increased detection and increased treatment rates on (a) total infected cases, (b) cirrhosis, (c) decompensated cirrhosis and (d) liver-related deaths. The green lines show rates with current rates of detection and treatment assuming use of the new therapies with their enhanced effectiveness. The grey lines show the effect of increasing diagnosis and treatment by 2.5 fold. Reproduced with permission. ${ }^{9} \mathrm{HCC}=$ hepatocellular carcinoma. 
that many patients are yet to be identified, the only practical way forward will be to treat patients who are identified as HCV positive as they are diagnosed, after the initial cohorts currently within secondary care have been treated.

\section{Possibilities of eradication}

Once the population known to services has been treated, the prospect of eradication of HCV is real. One key factor in achieving this would be to stop onward transmission and prevent new infections. This could be achieved by specifically targeting known high-risk groups, ie current injection drug users in substance misuse services or the prison population. An 8-12 week oral regimen with virtually no significant side effects is highly attractive in these populations in contrast to IFN-based therapies where uptake has been low. Modelling suggests that treatment will have a rapid and substantial impact on the spread of HCV in the injecting community. This effect could be very rapid if 'super-transmitters' can be targeted: individuals who may participate in injecting circles.

However, the key area for the future is case finding in primary care. People with a risk factor for HCV can be identified from primary care codes (eg for methadone prescribing, previous blood products, ethnicity and abnormal transaminase values) although the precise yield from these strategies is unknown in the UK population.

\section{Conclusion}

Modelling indicates that the abolition of HCV-related cirrhosis and mortality by 2030 is possible, but will overall require a more than doubling of both detection of new cases and of treatment rates (Fig 2). ${ }^{8,9}$

The advent of new drugs to treat hepatitis $C$ has opened up enormous opportunities to avoid premature mortality and morbidity from liver disease. The constraints of both our current service structures and the cost of therapy impose limitations on our ability to respond but the potential is there and it is now up to clinical teams to respond.

\section{References}

1 Penin F, Dubuisson J, Rey FA, Moradpour D, Pawlotsky JM. Structural biology of hepatitis C virus. Hepatology 2004;39:5-19.

2 Woerz I, Lohmann V, Bartenschlager R. Hepatitis C virus replicons: dinosaurs still in business? J Viral Hepat 2009;16:1-9.

3 Liang TJ, Ghany MG. Therapy of hepatitis C-back to the future. N Engl J Med 2014;370:2043-7.

4 Sulkowski MS, Gardiner DF, Rodriguez-Torres M et al. Daclatasvir plus sofosbuvir for previously treated or untreated hepatitis C infection. N Engl J Med 2014;370:211-21.

5 Harris H. Hepatitis C in the UK: 2014 report. London: Public Health England, 2014. Available online at www.gov.uk/government/uploads/ system/uploads/attachment_data/file/337115/HCV_in_the_ UK_2014_24_July.pdf [Accessed 26 January 2015].

6 Harris RJ, Hope VD, Morongiu A, Hickman M, Ncube F, DE Angelis D. Spatial mapping of hepatitis $C$ prevalence in recent injecting drug users in contact with services. Epidemiol Infect 2012;140:1054-63.

7 Martin NK, Foster GR, Vilar J et al. HCV treatment rates and sustained viral response among people who inject drugs in seven UK sites: real world results and modelling of treatment impact. J Viral Hepat 2014 Oct 7, epub ahead of print.

8 Harris RJ, Thomas B, Griffiths J et al. Increased uptake and new therapies are needed to avert rising hepatitis C-related end stage liver disease in England: modelling the predicted impact of treatment under different scenarios. J Hepatol 2014;61:530-7.

9 Cramp ME, Rosenberg WM, Ryder S, Blach S, Parkes J. Modelling the impact of improving screening and treatment of chronic hepatitis $C$ virus infection on future hepatocellular carcinoma rates and liver-related mortality. BMC Gastroenterol 2014;14:137.

Address for correspondence: Dr SD Ryder, Nottingham University Hospitals NHS Trust and Biomedical Research Unit, Queens Medical Centre, Nottingham, NG7 2UK, UK. Email: stephen.ryder@nuh.nhs.uk 